\title{
ON POWERS OF LIKELIHOOD FUNCTIONS OF RANDOM WALKS ON $\mathbb{Z}^{d}$
}

\author{
BY KRISHNA B. ATHREYA
}

\begin{abstract}
Let $\left\{X_{i}\right\}_{i>1}$ be independent, identically distributed random vectors in $\mathbb{Z}^{d}, d \geq 1$. Let $L_{n}(x) \equiv \mathbb{P}\left(S_{n}=x\right), n \geq 1, x \in \mathbb{Z}^{d}$, be the likelihood function for $S_{n}=\sum_{i=1}^{n} X_{i}$. For integers $j \geq 2$ and $n \geq 1$, let $a_{n}(j) \equiv \sum_{x \in \mathbb{Z}^{d}}\left(L_{n}(x)\right)^{j}$. We show that if $X_{1}-X_{2}$ has a nondegenerate aperiodic distribution in $\mathbb{Z}^{d}$ and $\mathbb{E}\left(\left\|X_{1}\right\|^{2}\right)<\infty$, then $\lim _{n \rightarrow \infty} n^{(j-1) d / 2} a_{n}(j) \equiv a(j, d)$ exists and $0<a(j, d)<\infty$. Some extensions and open problems are also outlined.

Keywords: Likelihood function; random walk; powers

2010 Mathematics Subject Classification: Primary 60E; 60G

Secondary $62 \mathrm{E}$
\end{abstract}

\section{Introduction}

In the classic book Aufgaben und Lehrsätze aus der Analysis [4] by Pólya and Szegö, the following result appears. For positive integers $j$ and $n, j \geq 2$,

$$
\sum_{k=0}^{n}\left(\begin{array}{l}
n \\
k
\end{array}\right)^{j} 2^{-n j} \sim j^{-1 / 2}\left(2 \pi n \cdot \frac{1}{4}\right)^{-(j-1) / 2},
$$

where ' $\sim$ ' denotes that the ratio of the two sides goes to 1 as $n \rightarrow \infty$. Farmer and Leth [3] use the central limit theorem to prove this result.

In a recent paper, the author and Janicki [1] established the following generalizations of (1) on powers of binomial and multinomial probabilities.

Binomial Case. Fix $0<p<1, q=1-p$. Then, for any integer $j \geq 2$, as $n \rightarrow \infty$,

$$
n^{(j-1) / 2} \sum_{k=0}^{n}\left[\left(\begin{array}{l}
n \\
k
\end{array}\right) p^{k} q^{n-k}\right]^{j} \rightarrow\left(j(2 \pi p q)^{j-1}\right)^{-1 / 2} .
$$

Multinomial Case. Fix $0<p_{1}, p_{2}, \ldots, p_{k}<1$, with $\sum_{i=1}^{k} p_{i}=1$. For integers $j \geq 2$ and $n \geq 1$, define

$$
b_{n, j, k}\left(p_{1}, p_{2}, \ldots, p_{k}\right) \equiv \sum_{\left(n_{1}, n_{2}, \ldots, n_{k}\right) \in T_{n, k}}\left[\left(\begin{array}{c}
n \\
n_{1}, n_{2}, \ldots, n_{k}
\end{array}\right) p_{1}^{n_{1}} \ldots p_{k}^{n_{k}}\right]^{j},
$$

where

$$
T_{n, k} \equiv\left\{\left(n_{1}, \ldots, n_{k}\right): 0 \leq n_{i} \leq n, \sum_{i=1}^{k} n_{i}=n\right\}
$$

Then, as $n \rightarrow \infty$,

$$
n^{(k-1)(j-1) / 2} b_{n, j, k}\left(p_{1}, \ldots, p_{k}\right) \rightarrow j^{-(k-1) / 2}(2 \pi)^{-(k-1)(j-1) / 2}\left(p_{1} \ldots p_{k}\right)^{-(j-1) / 2} .
$$


In this paper we generalize this result to study the asymptotics of integer powers of the likelihood function of random walks on $\mathbb{Z}^{d}$, the integer lattice in dimension $d \geq 2$. This is the content of Section 2. Some further extensions are outlined in Section 3 and some open problems are indicated in Section 4.

\section{Powers of likelihood functions of random walks}

In this section we establish the following result.

Theorem 1. Let $\left\{X_{i}\right\}_{i \geq 1}$ be independent, identically distributed (i.i.d.) random vectors in $\mathbb{Z}^{d}$, $d \geq 1$. Let $S_{0}=0$ and $S_{n}=\sum_{i=1}^{n} X_{i}, n \geq 1$. Let $L_{n}(x) \equiv \mathbb{P}\left(S_{n}=x\right), x \in \mathbb{Z}^{d}$, be the likelihood function of $S_{n}$. For integers $j \geq 2$ and $n \geq 1$, let

$$
a_{n}(j) \equiv \sum_{x \in \mathbb{Z}^{d}}\left[L_{n}(x)\right]^{j} .
$$

Suppose that $\mathbb{E}\left(\left\|X_{1}\right\|^{2}\right)<\infty$ and that $X_{1}-X_{2}$ has a nondegenerate aperiodic distribution in $\mathbb{Z}^{d}$. Then, for each integer $j \geq 2$, the limit

$$
\lim _{n \rightarrow \infty} n^{(j-1) d / 2} a_{n}(j) \equiv a(j, d)
$$

exists, and $0<a(j, d)<\infty$.

Proof. Let $\left\{S_{n i}\right\}_{n \geq 0}, i=1,2, \ldots$ be i.i.d. random walks with the same distribution as the random walk $\left\{S_{n}\right\}_{n \geq 0}$ defined in the statement of the theorem. Then

$$
\begin{aligned}
\mathbb{P}\left(S_{n 1}=S_{n 2}=\cdots=S_{n j}\right) & =\sum_{x \in \mathbb{Z}^{d}} \mathbb{P}\left(S_{n 1}=S_{n 2}=\cdots=S_{n j}=x\right) \\
& =\sum_{x \in \mathbb{Z}^{d}} \mathbb{P}\left(S_{n 1}=x\right) \mathbb{P}\left(S_{n 2}=x\right) \cdots \mathbb{P}\left(S_{n j}=x\right) \\
& =\sum_{x \in \mathbb{Z}^{d}}\left[L_{n}(x)\right]^{j}
\end{aligned}
$$

by independence and the fact that the $\left\{S_{n i}\right\}_{n \geq 0}, i=1,2, \ldots$ are i.i.d. random walks with the same distribution as $\left\{S_{n}\right\}_{n \geq 0}$. Thus, for $j \geq 2$ and $n \geq 0$,

$$
\begin{aligned}
a_{n}(j) & =\mathbb{P}\left(S_{n 1}=S_{n 2}=\cdots=S_{n j}\right) \\
& =\mathbb{P}\left(S_{n 1}-S_{n r}=\mathbf{0}_{d}, r=2, \ldots, j\right) \\
& =\mathbb{P}\left(\sum_{i=1}^{n} Y_{i}=\mathbf{0}_{(j-1) d}\right),
\end{aligned}
$$

where $\mathbf{0}_{n}$ is the zero vector in $\mathbb{Z}^{n}$ and $\left\{Y_{i}\right\}_{i \geq q}$ are i.i.d. random vectors distributed as $Y_{1}=$ $\left(X_{1}-X_{2}, X_{1}-X_{3}, \ldots, X_{1}-X_{j}\right)$. By construction, $Y_{1}$ has mean $\mathbf{0}_{(j-1) d}$, and $\mathbb{E}\left(\left\|Y_{1}\right\|^{2}\right)<\infty$, where $\|\cdot\|$ denotes the Euclidean norm on $\mathbb{R}^{(j-1) d}$, and $Y_{1}$ has a nondegenerate aperiodic distribution in $\mathbb{Z}^{(j-1) d}$.

Assertion (2) now follows from the local central theorem for lattice-valued random vectors [2], [5]. Furthermore, $a(j, d)$ can be identified with

$$
\frac{1}{(2 \pi)^{(j-1) d / 2}|\Sigma|^{1 / 2}},
$$

where $\Sigma$ is the variance-covariance matrix of the random vector $Y_{1}$, and this is finite and positive. 


\section{A further extension}

For $j \geq 2$, fix positive integers $r_{2}, \ldots, r_{j}$. Let

$$
T\left(r_{2}, \ldots, r_{j}\right)=\left\{\boldsymbol{x}=\left(x_{1}, \ldots x_{j}\right): x_{i} \in \mathbb{Z}^{d},\left|x_{k}-x_{1}\right|<r_{k}, 2 \leq k \leq j\right\},
$$

where, for $\boldsymbol{v}=\left(v_{1}, \ldots, v_{d}\right) \in \mathbb{Z}^{d}$ with $v_{i} \in \mathbb{Z},|\boldsymbol{v}|=\sum_{i=1}^{d}\left|v_{i}\right|$. Let

$$
a_{n}\left(j, r_{2}, \ldots r_{j}\right) \equiv \sum_{\left(x_{1}, \ldots, x_{j}\right) \in T\left(r_{2}, \ldots, r_{j}\right)} L_{n}\left(x_{1}, x_{2}, \ldots, x_{j}\right) .
$$

As in the proof of Theorem 1, we can identify the right-hand side of (4) with

$$
\begin{aligned}
& \mathbb{P}\left(\left|S_{n 1}-S_{n 2}\right| \leq r_{2}, \ldots,\left|S_{n 1}-S_{n j}\right| \leq r_{j}\right) \\
& =\sum_{\left|y_{2}\right| \leq r_{2}, \ldots,\left|y_{j}\right| \leq r_{j}} \mathbb{P}\left(S_{n 1}-S_{n 2}=y_{2}, \ldots, S_{n 1}-S_{n j}=y_{j}\right) .
\end{aligned}
$$

By Theorem 1, for each $\left(y_{2}, \ldots, y_{j}\right)$, as $n \rightarrow \infty$,

$$
n^{(j-1) d / 2} \mathbb{P}\left(S_{n 1}-S_{n 2}=y_{2}, \ldots, S_{n 1}-S_{n j}=y_{j}\right) \rightarrow a(j, d) .
$$

Now the cardinality of $\left\{\left(y_{2}, \ldots, y_{j}\right) \in \mathbb{Z}^{d(j-1)}:\left|y_{i}\right| \leq r_{i}\right\}$ equals $\left(2 r_{2}+1\right)\left(2 r_{3}+1\right) \cdots\left(2 r_{j}+1\right)$, so we have the following result.

Theorem 2. Let $a_{n}\left(j, r_{2}, \ldots, r_{j}\right)$ be as in (4). Then, as $n \rightarrow \infty$,

$$
n^{\frac{1}{2}(j-1) d} a_{n}\left(j, r_{2}, \ldots, r_{j}\right) \rightarrow a(j, d)\left(2 r_{2}+1\right) \cdots\left(2 r_{j}+1\right),
$$

where $a(j, d)$ is as in (3) in Theorem 1.

\section{Some open problems}

Problem 1. Theorem 1 assumes that $j$ is an integer greater or equal to 2 . The main tool used in its proof is the identification of $a_{n}(j)$ with the probability that an aperiodic random walk in a lattice of dimension $\mathbb{Z}^{(j-1) d}$ with zero-mean step visits $\mathbf{0}$ at step $n$, followed by an appeal to a local central limit theorem. For noninteger $j$, this tool is not available, but the question is still of interest.

Problem 2. A special case of the problem above is to understand the rate of decay (or growth) (as $n \rightarrow \infty$ ) of

$$
\sum_{k=0}^{n}\left[\left(\begin{array}{l}
n \\
k
\end{array}\right) p^{k} q^{n-k}\right]^{j}
$$

for noninteger $0<j<\infty$.

Problem 3. (Nonlattice case.) Let $\left\{X_{i}\right\}_{i \geq 1}$ be i.i.d. random vectors in $\mathbb{R}^{d}$ for some $d \geq 1$. Let $L_{n}(x)$ be the probability density function of $S_{n}=\sum_{i=1}^{n} X_{i}$ with respect to some reference measure $\mu$. What is the rate of decay (for $1<j<\infty$ ) or growth (for $0<j<1$ ) as $n \rightarrow \infty$ of

$$
\int_{\mathbb{R}^{d}}\left[L_{n}(x)\right]^{j} \mu(\mathrm{d} x) ?
$$

Problem 4. The same problem as above for the case in which $X_{1}-X_{2}$ does not have finite second moment. Some result analogous to the local central limit theorem for random walks whose i.i.d. steps have mean zero but infinite second moment is needed here. 


\section{References}

[1] Athreya, K. B. AND Janicki, R. (2016). Asymptotics of powers of binomial and multinomial probabilities. Statist. Prob. Lett. 112, 58-62.

[2] Davis, B. And McDonald, D. (1995). An elementary proof of the local central limit theorem. J. Theoret. Prob. 8, 693-701.

[3] FARmer, J. D. ANd Leth, S. C. (2005). An asymptotic formula for powers of binomial coefficients. Math. Gazette 89, 385-391.

[4] Pólya, G. And Szegö, G. (1945). Aufgaben und Lehrsätze aus der Analysis. Dover, New York (in German).

[5] SPITZer, F. (1976). Principles of Random Walk (Graduate Texts Math. 34), 2nd edn. Springer, New York.

KRISHNA B. ATHREYA, Iowa State University

Department of Mathematics and Department of Statistics, Iowa State University, Ames, IA, USA.

Email address: kbathreya@gmail.com 